\title{
Adjoint zero-modes as a tool to understand the Yang-Mills vacuum
}

\section{Margarita García Pérez}

Instituto de Física Teórica UAM-CSIC

Universidad Autónoma de Madrid, Cantoblanco, 28049 Madrid, Spain

E-mail: margarita.garcia@uam.es

\section{Antonio González-Arroyo}

Departamento de Física Teórica and Instituto de Física Teórica UAM-CSIC

Universidad Autónoma de Madrid, Cantoblanco, 28049 Madrid, Spain

E-mail: antonio.gonzalez-arroyo@uam.es

\author{
Alfonso Sastre* \\ Departamento de Física Teórica and Instituto de Física Teórica UAM-CSIC \\ Universidad Autónoma de Madrid, Cantoblanco, 28049 Madrid, Spain \\ E-mail: alfonso.sastre@uam.es
}

\begin{abstract}
The use of adjoint (quasi) zero-modes of the Dirac operator to probe the Yangs-Mills vacuum has been recently advocated by González-Arroyo and Kirchner. The construction relies on the use of the super-symmetric zero mode which, for classical configurations, provides a direct estimate of the gauge action density. In the lattice implementation of this idea, we show how the results improve considerably if the overlap operator is used instead of the Wilson-Dirac one. Before proceeding to the detailed study of Monte Carlo ensembles, we studied here a series of potentially complicated situations which can be encountered. In particular, we study the case of instanton anti-instanton pairs and analyse how the results depend upon separation. The effect of lattice artifacts is also of concern. Indeed, a statistical analysis of zero modes of thermalised SU(2) configurations at $\beta=2.57$ shows a significant fraction having $4 N+2$ adjoint zero modes, in contradiction with the index theorem. This violation must be associated to the roughness of the lattice configurations. Indeed, we show that this situation occurs for instantons of size of the order of the lattice spacing.
\end{abstract}

The XXV International Symposium on Lattice Field Theory

July 30 - August 42007

Regensburg, Germany

\footnotetext{
${ }^{*}$ Speaker.
} 


\section{Introduction.}

In the past years a considerable effort has been devoted to the analysis of the topological structure of pure Yangs-Mills theory on the lattice. These studies encompass from global quantities, like the topological susceptibility, to local ones, in an attempt to link topology to models of chiral symmetry breaking and confinement. For that one has to deal with the roughness of lattice MonteCarlo configurations, which are generally plagued with ultraviolet fluctuations. Several cooling and smearing algorithms have been proposed in order to obtain a smoother image of these configurations. Although these methods are useful in order to compute global quantities, they are criticized as a tool to analyse local properties. An alternative, claimed to distort less the original ensemble, is to use filtering methods based on the Dirac operator [1]. The main idea is the relation between fermions and topology given by the Atiyah-Singer index theorem and the correlation between the gauge action density and the local density of the eigenstates of the Dirac operator. As will be described below, this relation is particularly neat for fermions in the adjoint representation.

In what follows we will first present a discussion of the advantages of using filtering methods based on the adjoint Dirac operator, as advocated in [2]. In that paper it was argued that in order to apply the method to Monte Carlo generated configurations on the lattice the use of the overlap Dirac operator was desirable. Here we have carried out this program and applied it to a set of potentially delicate situations. The first one is that of instanton anti-instanton (IA) pairs. The construction in [2] is exact for classical solutions of the equations of motion and relies on the existence of quasi zero modes of the Dirac operator. IA pairs are not of this sort, with quasi zero modes disappearing as the separation in the pair decreases. Still our results show how, even in the case of rather small separation, the topological charge density can be reconstructed in terms of the density of the, so called, super-symmetric modes. The second potentially delicate case is related to dislocations, lattice artefacts associated to small instantons. Some time ago Edwards, Heller and Narayanan [3] reported on the presence of configurations with $4 N+2$ adjoint-zero modes in lattice thermalized ensembles. This is in contrast with the continuum expectation of $4 N$. We have also encountered a significant fraction of these cases in our ongoing Monte-Carlo simulations. We will argue that they are necessarily related to the roughness of the lattice configurations and show it explicitly on a set of instantons with size of the order of the lattice spacing.

\section{Reconstructing the action density from adjoint zero-modes.}

We will describe here the basic ideas underlying the proposal in Ref. [2] and what are the advantages over other filtering methods.

The construction is based on the super-symmetric zero modes for classical solutions of the Euclidean equations of motion, which read [4]:

$$
\psi^{a}=\frac{1}{8} F_{\mu \nu}^{a}\left[\gamma_{\mu}, \gamma_{v}\right] V
$$

with $F_{\mu \nu}$ the gauge field strength and $V$ any constant four-spinor. From this expression two positive and two negative chirality zero modes are obtained (note that adjoint zero modes always come in pairs related by charge conjugation $C$ through $\left.\psi_{c}=\gamma_{5} C \psi^{*}\right)$. The densities of these zero modes 
give respectively the self-dual and anti-self-dual parts of the action density. Taking, for instance, $V^{+}=(1,0,0,0)$, the corresponding positive chirality zero mode in Eq. (2.1) becomes

$$
\psi_{+}^{a}(x) \propto\left(\begin{array}{c}
E_{3}^{a}(x)+B_{3}^{a}(x) \\
E_{1}^{a}(x)+B_{1}^{a}(x)-i\left(E_{2}^{a}(x)+B_{2}^{a}(x)\right) \\
0 \\
0
\end{array}\right),
$$

selecting only the self-dual part of the action density. There is one peculiarity of $\psi_{+}^{a}$ that allows to single it out from the subspace of zero modes: the imaginary part of the first component is zero in every point $x$ and for all components in colour space.

Given the fact that, for classical solutions, the super-symmetric zero modes trace the action density, the proposal in Ref. [2] is to make an analogous construction on generic configurations. The strategy is to look for the low lying modes of the Dirac operator and single out the supersymmetric modes by imposing reality conditions as the one described above. For example, within the space of positive chirality low modes we select the combination that minimizes the quantity $\sum_{x, a}\left[\operatorname{Im} \psi_{+}^{1, a}(x)\right]^{2}$. Charge and action densities are reconstructed respectively out of the sum and difference of $\left|\psi_{+}^{a}(x)\right|^{2}$ and $\left|\psi_{-}^{a}(x)\right|^{2}$. This method is computationally cheaper than those based on the use of the fundamental representation, since it requires a much smaller number of modes to reconstruct the (anti) self-dual structures.

The authors in Ref. [2] have tested the method using the Wilson-Dirac operator. The test works well on smooth configurations. Once moderate quantum fluctuations are included the method succeeds in filtering the UV noise. Nevertheless, the Wilson-Dirac operator is not optimal for this program since, in many cases, it does not show a clear gap in the spectrum. A better option is to use the Neuberger-Dirac operator [5]:

$$
D_{o v}=\frac{1}{2}\left(1+\gamma_{5} \varepsilon\left(H_{W D}\right)\right),
$$

where $H_{W D}=\gamma_{5}\left(D_{W}-m_{W D}\right)$ and $D_{W}$ is the Wilson-Dirac operator in the adjoint representation. One advantage of using a chiral operator is that we can compute each chirality independently by diagonalising the operators:

$$
D \bar{D}=P_{+}\left(\gamma_{5} D_{o v}\right)^{2} P_{+}=\frac{1}{2} P_{+}(1+\varepsilon) P_{+}
$$

and

$$
\bar{D} D=P_{-}\left(\gamma_{5} D_{o v}\right)^{2} P_{-}=\frac{1}{2} P_{-}(1-\varepsilon) P_{-}
$$

with $P_{ \pm}=\frac{1}{2}\left(1 \pm \gamma_{5}\right)$.

As mentioned in the introduction we will test the method on particularly complicated situations, including non-self dual configurations and small instantons.

\section{Testing the method on instanton-anti-instanton pairs.}

Instanton - anti-instanton (IA) pairs are non-self-dual configurations with zero topological charge. Although such configurations do not have exact zero modes, we expect to find quasi 


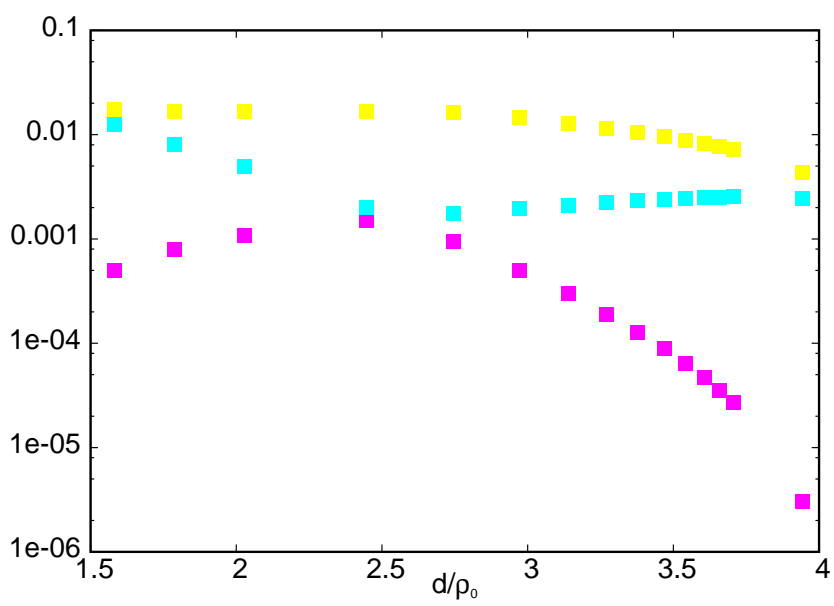

Figure 1: Spectrum of lower modes of an IA pair. Each point represents two positive and two negative chirality modes. For large separation the super-symmetric modes are the lowest (pink) modes in the spectrum. This ceases to be the case after the level crossing at $d \sim 2.5 \rho_{0}\left(\rho_{0}=3 a\right.$ is the original instanton size).

Negative chirality

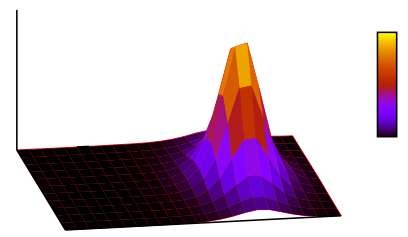

$\mid P$ si $\left._{+}\right|^{2}-\mid P$ si._. $^{2}$

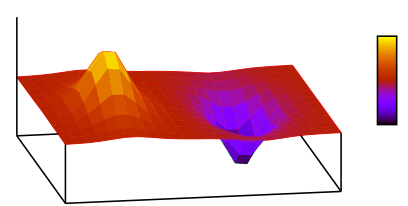

Positive chirality

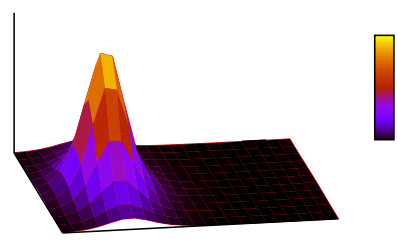

Charge density

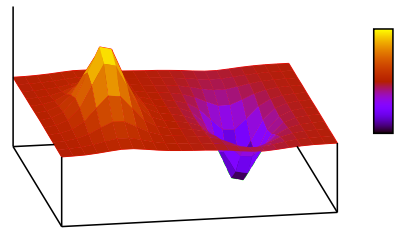

Figure 2: Top: Positive and negative chirality lowest modes for a well separated instanton-anti-instanton pair. Bottom: Reconstruction of the charge density out of the lowest modes compared with the original one.

zero-modes for well separated pairs. In particular, we expect 4 positive and 4 negative chirality zero-modes associated to the isolated instanton and anti-instanton. Indeed, our results for large separation, presented in Fig. 1, show a clear gap between the lowest eigenvalues and the rest of the spectrum. The degeneracy of these lowest modes is, however, not correct. We find only two positive and two negative chirality solutions corresponding, at large separation, to the super-symmetric zero-modes. Figure 2 shows the densities of these modes. Despite the discrepancy in the degeneracy of modes, the self-dual and anti-self dual parts of the action density can be extracted with good accuracy and the reconstruction of the original charge density turns out to be very good.

The situation becomes less clear as the instanton and anti-instanton begin to overlap strongly (a 


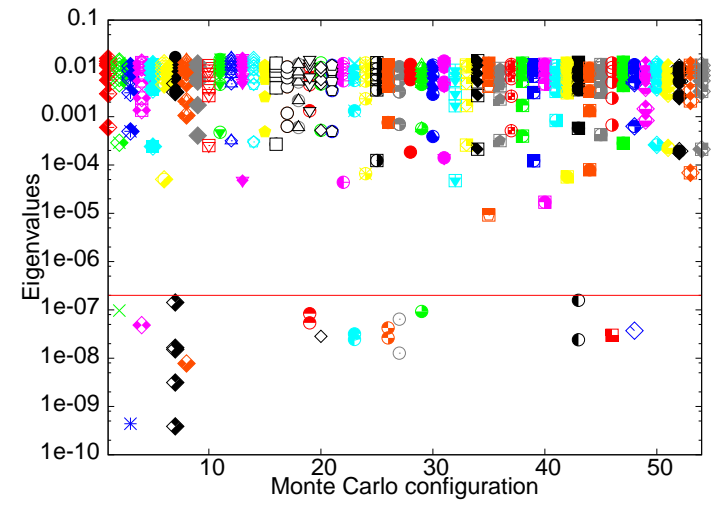

Figure 3: Spectra of $D_{o v}\left(m_{W D}=1.4\right)$ for the Monte Carlo configurations. Points below the straight line are compatible with zero.

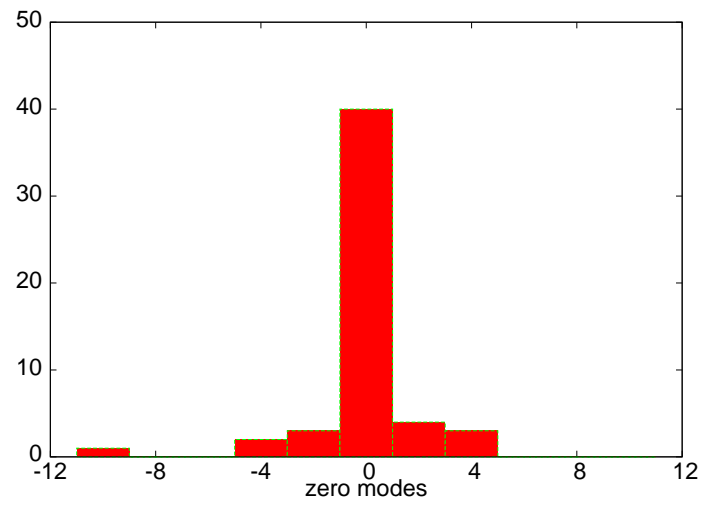

Figure 4: Histogram of the number of configurations with a given number of zero modes. Negative number corresponds to negative chirality.

sequence of configurations at different separations can be generated by improved cooling [6]). In Fig. 1, we can see how the lowest eigenvalues grow as the separation decreases. There is a critical distance $\left(d=2.5 \rho_{0}\right)$ where the gap in the spectrum closes. In addition to this, there is level crossing and the super-symmetric modes cease to be the lowest ones in the spectrum. Nevertheless, even in such case, the super-symmetric modes reconstruct the charge density profile in good agreement with the original one.

\section{Testing the method on rough configurations.}

As an ongoing part of the project, we have started analyzing configurations extracted from a SU(2) thermalized ensemble. They have been generated with the Wilson plaquette action, on a $12^{4}$ lattice for $\beta=2.57$ ( $a=0.08 \mathrm{fm}$ ). For fifty Monte-Carlo configurations we have extracted the twelve lowest eigenvalues and eigenvectors. The eigenvalues are collected in Fig. 3, showing a clear gap between zero and non-zero modes. A histogram of the number of zero modes is presented in Fig. 4. Surprisingly a significant fraction of the configurations shows only two zero modes, while the continuum index theorem predicts $4 N, N \in \mathbb{Z}$. As mentioned before, this mismatch was previously reported by Edwards, Heller and Narayanan [3], who interpreted it as evidence for fractional topological charge (not expected on the continuum for the periodic boundary conditions used). Contrary to their belief, we will argue that this effect is an artefact associated to the roughness of the lattice configurations. We will show that in some cases it can be related to the presence of small instantons with sizes of the order of the lattice spacing.

To analyse the behaviour of small instantons we start with a smooth $S U(2)$ configuration with $Q=1$. After applying to it several cooling sweeps with $\varepsilon=1$ we obtain a sequence of configurations for different instanton sizes. On them, we compute the eight first adjoint eigenvalues using $m_{W D}=1.4$ in the overlap operator, see Eq. (2.3). The Atiyah-Singer Index theorem predicts in this case four zero-modes, in two independents pairs $\left(\psi, \psi_{c}\right)$. Figure 5 shows the spectrum as a function of the instanton size. Indeed, the initial configuration has 4 zero-modes. However, there is a critical size $\left(\rho_{c}=2.05 a\right)$ below which only two zero modes remain. Fig. 6 shows the dependence of $\rho_{c}$ on the mass of the overlap operator. It is rather mild for $m_{W D}>1.4$. We can use this fact 


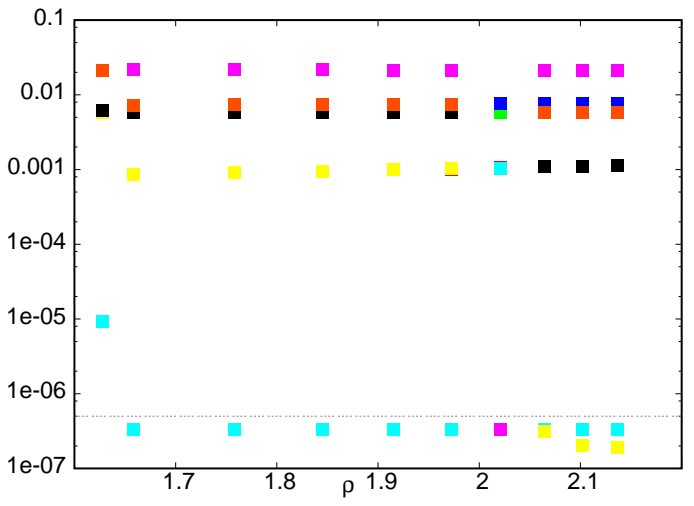

Figure 5: Spectrum of $D_{o v}\left(m_{W D}=1.4\right)$ versus the instanton size in lattice units. Points below the line are doubly degenerate and compatible with zero.

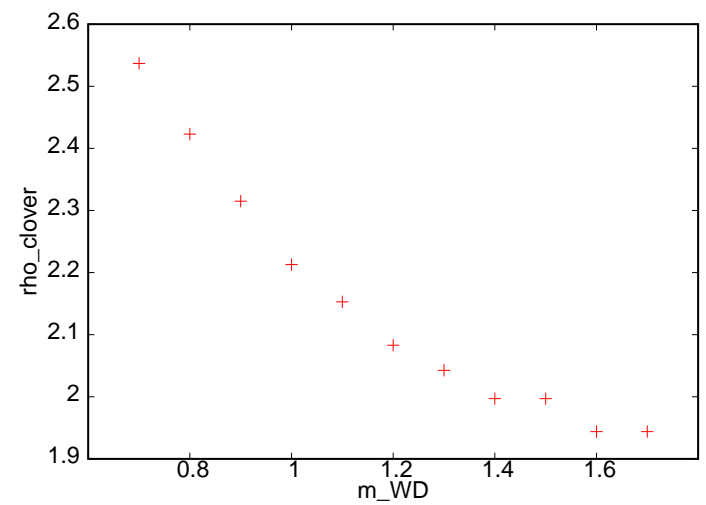

Figure 6: Dependence of $\rho_{c}$ on the mass of the overlap operator. $\rho_{c}$ is the critical size (in lattice units) below which the spectrum has 2 zero modes.

to select an optimal value of $m_{W D}$ for the analysis of the MC simulations. The two spurious zero modes dissappear for $\rho<1.65 a$, quite independently of the value of $m_{W D}$. Using $m_{W D}>1.4$, for which $\rho_{c} \sim 1.9 a$, minimises, hence, the window for spurious eigenvalues.

The spurious zero modes are clearly associated with the roughness of the configuration. This can be quantified through the admissibility condition that should be imposed in order to guarantee locality of the overlap operator [7]. This condition, written in terms of the plaquette $U(p)$, reads

$$
\|1-U(p)\| \leq \varepsilon\left(m_{W D}\right) .
$$

Figure 7 shows the number of plaquettes that violate Eq. 4.1, as a function of instanton size for the configurations presented in Fig. 6. There is a clear link between configurations with spurious zero modes and those that violate the admissibility condition. To stress it further we have analysed the location of 'wrong plaquettes'. Fig. 8 shows that they are localized around the maximum of the action density where the configuration becomes rougher for small instantons.

Note that this mismatch is irrelevant if the set of rough configurations has zero measure in the continuum limit. Our $\beta=2.57$ set has been generated with the SU(2) Wilson action. Indeed for this action, general arguments, due to Pugh and Teper [8], indicate a divergent contribution of small instantons in the continuum limit. The problem can be avoided by using an improved action for the Monte-Carlo generation and by tuning the mass in the overlap operator. Similar artefacts in the spectrum of the overlap Dirac operator in the fundamental representation have been reported in $[9,10]$. All this is an indication that the use of improved actions might be essential even for the computation of global quantities as the topological susceptibility.

\section{Conclusions.}

We have argued that the adjoint zero-modes of the Dirac operator provide an efficient way to extract the topological content of the Yang-Mills vacuum. The method requires the evaluation of a small numer of eigenvectors, reducing the computational cost wtih respect to approaches based on the fundamental representation. It makes use of the properties of a special set of zero modes which, for classical configurations, are directly linked to the gauge action density. We have probed 


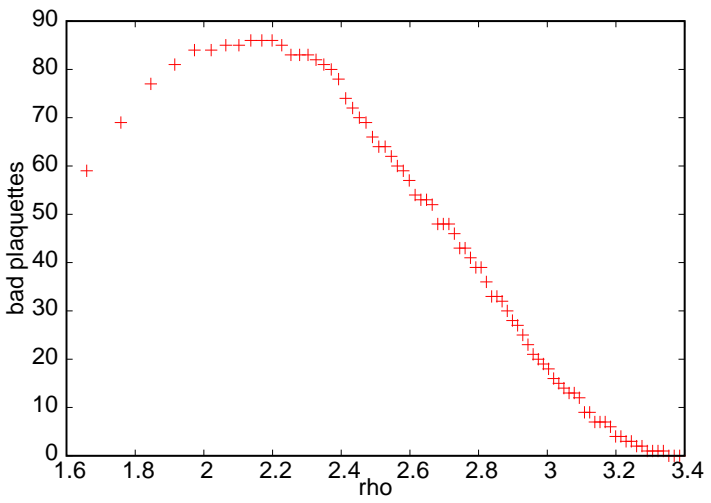

Figure 7: Number of plaquettes that violate the admissibility condition Eq. (4.1) in terms of the instanton size. $\varepsilon(1)=1 / 30$.

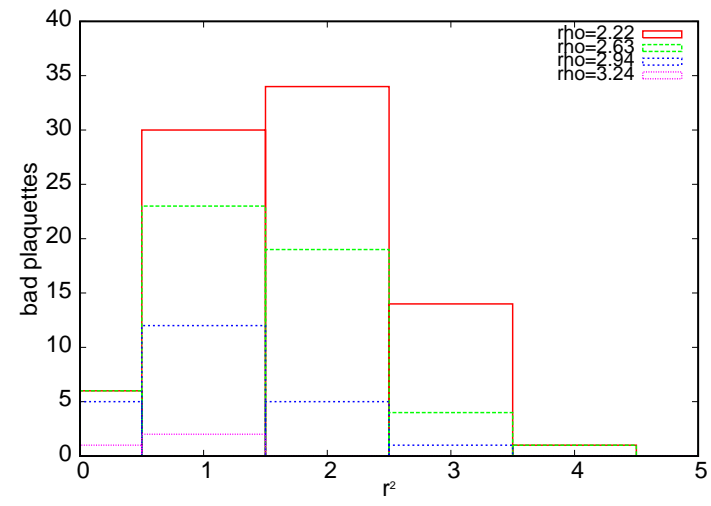

Figure 8: Number of plaquettes that violate the admissibility condition vs distance from the maximum of the action density, for several instanton sizes.

the goodness of the method in reproducing the charge density of, non-self-dual, instanton antiinstanton pairs. We have also analysed the case of rough configurations as small instantons. We have pointed out that a mismatch between the observed number of zero modes and the continuum prediction generically arises for instantons below a critical size of the order of the lattice spacing.

\section{Acknowledgments}

We acknowledge financial support from the Madrid Regional Government (CAM), under the program HEPHACOS P-ESP-00346, and the Spanish Research Ministry (MEC), under contracts FPA2006-05807, FPA2006-05485, FPA2006-05423. Also acknowledged is the use of the MareNostrum supercomputer at the BSC-CNS and the IFT-UAM/CSIC computation cluster.

\section{References}

[1] See e.g. F. Bruckmann's plenary talk in these proceedings and E. M. Ilgenfritz e.a., Phys. Rev. D 76 (2007) 034506 and references therein.

[2] A. Gonzalez-Arroyo and R. Kirchner, JHEP 0601 (2006) 029.

[3] R. G. Edwards, U. M. Heller and R. Narayanan, Phys. Lett. B 438 (1998) 96. Nucl. Phys. Proc. Suppl. 73 (1999) 497.

[4] S. Chadha, A. D'Adda, P. Di Vecchia and F. Nicodemi, Phys. Lett. B 67 (1977) 103. R. Jackiw and C. Rebbi, Phys. Rev. D 16 (1977) 1052.

[5] H. Neuberger, Phys. Lett. B 417 (1998) 141. H. Neuberger, Phys. Rev. Lett. 81 (1998) 4060.

[6] M. García Pérez, A. González-Arroyo, J. R. Snippe and P. van Baal, Nucl. Phys. B 413 (1994) 535.

[7] P. Hernandez, K. Jansen and M. Luscher, Nucl. Phys. B 552 (1999) 363.

[8] D. J. R. Pugh and M. Teper, Phys. Lett. B 224 (1989) 159.

[9] A. Hasenfratz, R. Hoffmann and S. Schaefer, "Localized eigenmodes of the overlap operator and their impact on the eigenvalue distribution," arXiv:0709.0932 [hep-lat].

[10] V. G. Bornyakov et.al. Phys. Rev. D 76 (2007) 054505. 